\title{
Microwave Drying of Textile Materials and Optimization of a Resonant Applicator
}

M. Pourová, J. Vrba

The principal aim of this work was to design and optimize the applicator for microwave drying. Our applicator is derived from the Fabry-Perrot resonator, which is an open type resonator. The whole system works at frequency 2.45 GHz and the magnetron that we used delivers power $800 \mathrm{~W}$. This machine is intended for use in drying in factory production of fabrics.

After identifying of the basic arrangement of the microwave drying machine, the next step in the design was the use of the electromagnetic field simulator. We determined the position of the magnetron and found the distribution of the electric field strength in drying textiles in this way. In parallel, we analyzed the drying system with analytical calculations. We created a diagram of the EM waves inside this structure and reached the resulting expression for use in calculating the strenght of the electric field in the plane of the drying textile. This quantity depends on the electrical characteristics of wet textiles, e.g. the permittivity and the loss factor. Measurements of these dielectric properties for the coburg is complicated, and this method makes it possible to solve our problem with dielectric parameters.

We have SAR distribution results (by simulation and also by measurement), results of measurements of the moisture content in the dried textile with respect to time. These results are important for subsequent optimization of the efficiency of the whole machine.

Keywords: Microwave drying, open resonator, drying of textile, SAR distribution.

\section{Notation}

SAR

$\rho_{1}$

$\rho_{2}$

$\sigma_{2}=\sqrt{1-\rho_{2}^{2}}$ transmission factor,

$e^{-x}=e^{-\alpha_{\text {tex }} t} \quad$ absorption in textile,

$\alpha_{\text {tex }} \quad$ attenuation factor of textile,

$t \quad$ thickness of the textile,

$\beta \quad$ phase constant of free space,

$\ell \quad$ distance between reflective plate and textile,

$\varepsilon_{\text {tex }} \quad$ relative permittivity,

$\operatorname{tg} \delta_{\text {tex }} \quad$ loss factor.

\section{Introduction}

Microwave heating is a very promising technology which has been finding new applications in industry. It is a technology which can replace conventional heating. We would like to describe our new microwave industrial applicator used for drying textiles in manufacturing. In this drying process, a very thin layer of textile material does not have a very well defined position in the applicator. Also the complex permittivity of dried textile is not constant during the procedure. Its value changes in time with respect to the decreasing moisture content [1].

We designed and analyzed our open-resonator applicator. In this paper we will present the theoretical model, the results of EM field and SAR numerical modeling, and experimental evaluation. The prototype of the microwave drying machine working at frequency $2.45 \mathrm{GHz}$, also is created from 17 cells, will be described here.

\section{Design of the applicator}

Our new microwave industrial applicator for drying textiles is based on the principle of the Fabry-Perrot resonator, which is an open type resonator. This applicator has a magnetron as a source of high electromagnetic power, placed in the waveguide holder. The power of the magnetron is $800 \mathrm{~W}$ and its working frequency is $2.45 \mathrm{GHz}$. Dried textile material is located in the middle plane between the parallel conductive plates, and the distance between these plates is equal to (3/2) $\lambda$ [2]. Fig. 1 shows the scheme of this applicator.

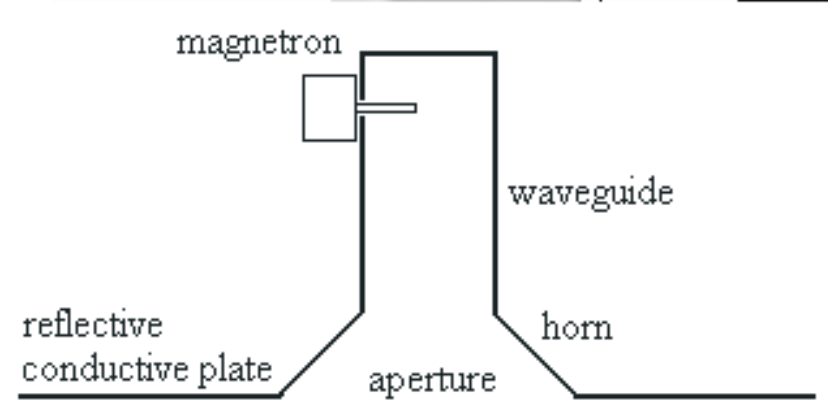

textile

reflective conductive plate

Fig. 1: Scheme of the open-resonator type applicator

In the first part of design we used a simulator of the electromagnetic field. We determined the position of the magnetron, and in this way we found the distribution of the electric field strength in the drying textile. Fig. 2a shows the impedance matching of the systems that were obtained by 


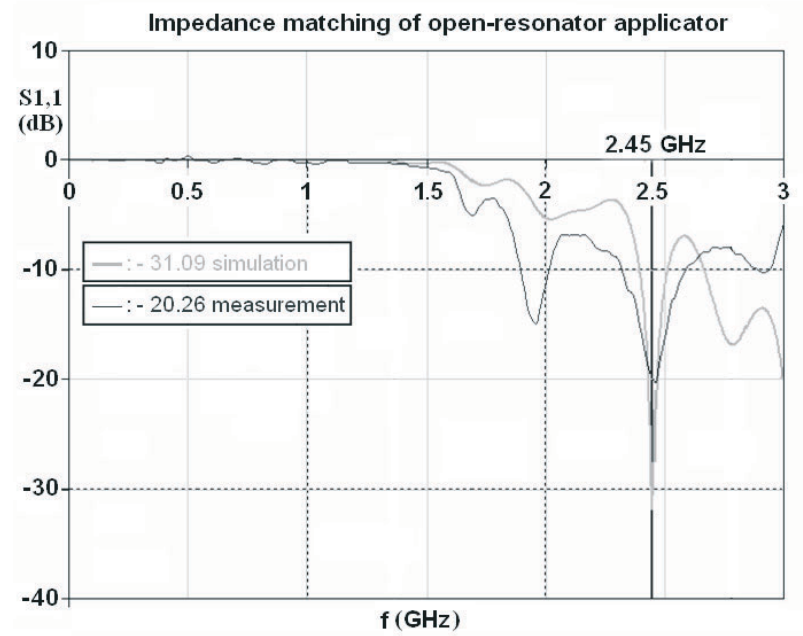

(a)
Impedance matching of open-resonator applicator

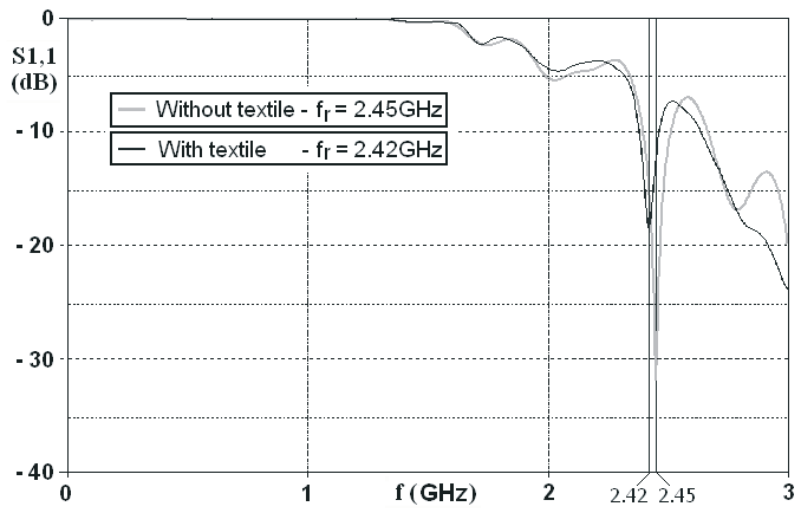

(b)

Fig. 2: Impedance matching of the applicator

simulation and by measurement. The resonant frequency is changed if we simulate the applicator with textile (Fig. 2b). However, this applicator can be tuned back to resonance by changing the position of the parallel plates.

Fig. 3a shows the simulated distribution of the electric field strength in the applicator for textile with complex per-
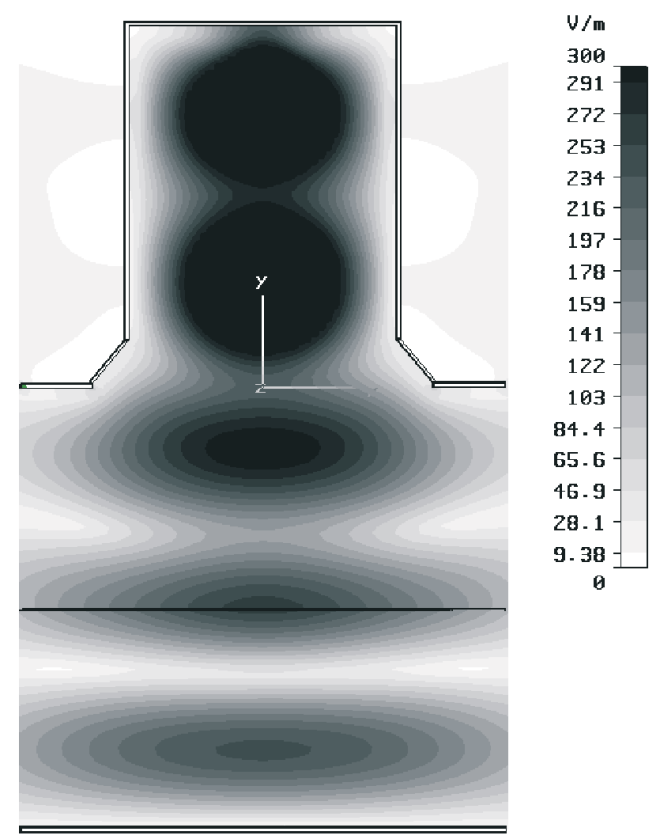

(a)

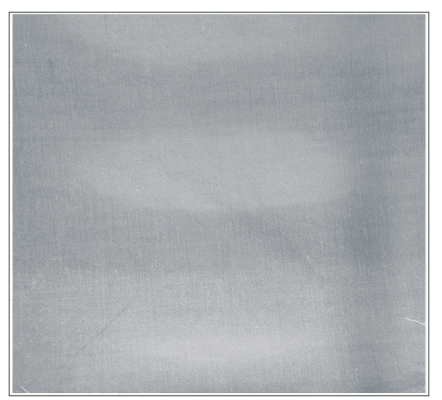

(b)

Fig. 3: Distribution of electric field strength (a) and an example of a drying experiment (b) mittivity 8 and loss factor 0.566 . The thickness of the textile used here is $0.3 \mathrm{~mm}$ and the weight is $210 \mathrm{~g} / \mathrm{m}^{2}$. Fig. 4a shows the SAR characteristic calculated with simulation software. Fig. 3b and 4b show examples of the drying experiments. Both processes are different, but they give very well comparable results.

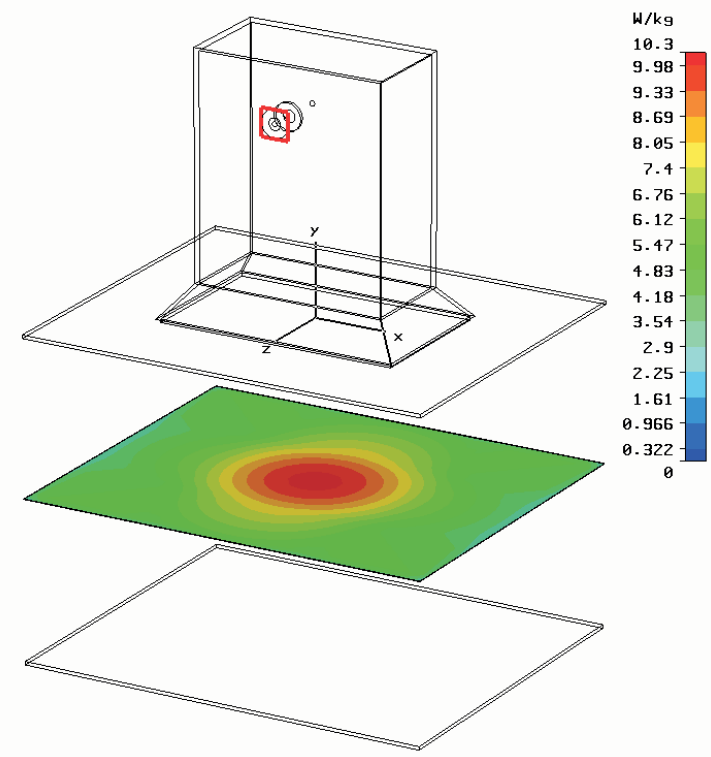

(a)

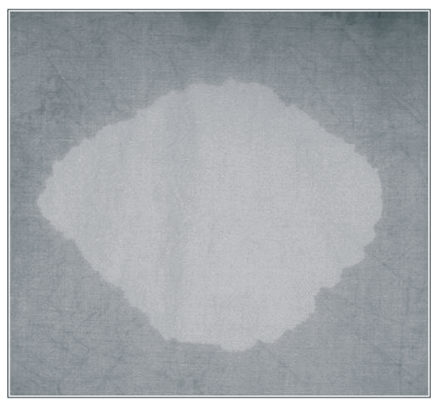

(b)

Fig. 4: SAR distribution in textile (a) and an example of a drying experiment (b) 


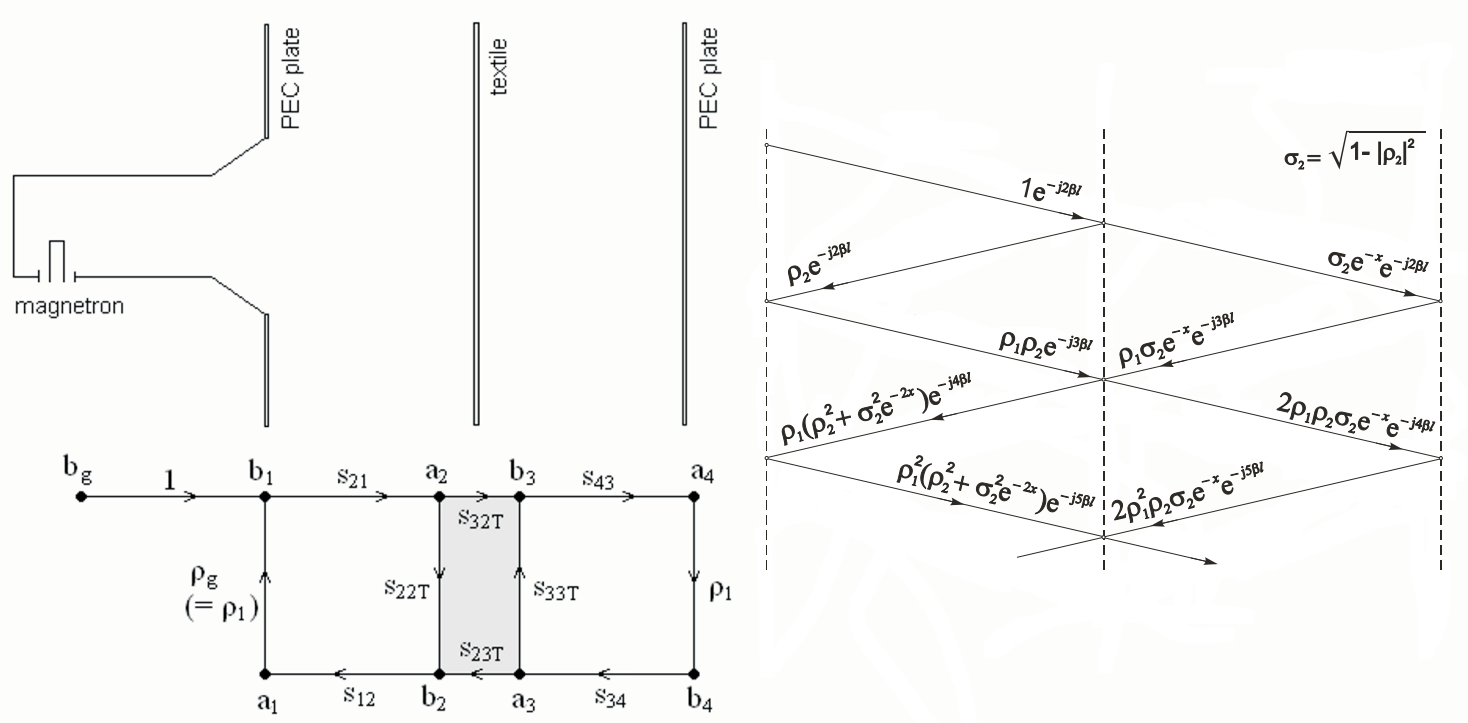

Fig. 5: Tools for optimizing the microwave dryer

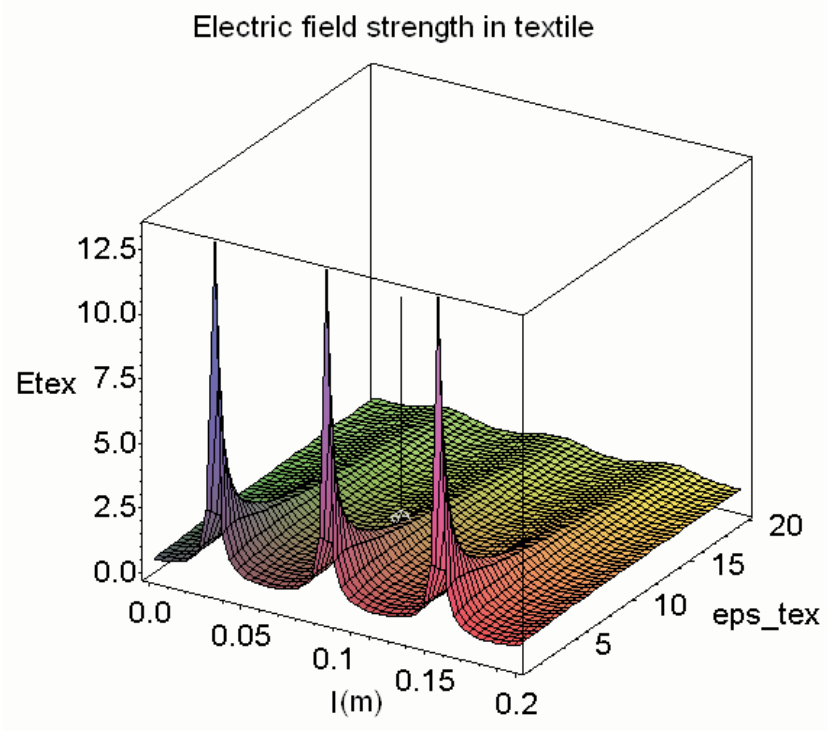

a)

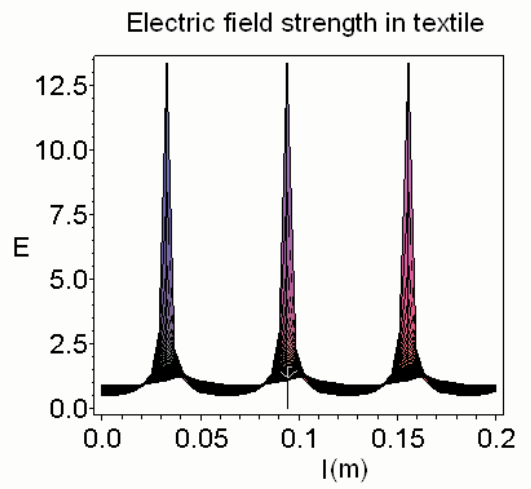

b)

Fig. 6: Dependence of electric field strength on distance $\ell$ and relative permittivity $\varepsilon_{\text {tex }}(\operatorname{tg} \delta=0.566), 3 \mathrm{D}$ (a) and $2 \mathrm{D}$ (b) display of the quantities

\section{Optimization of the resonant applicator}

Our drying resonant system is optimized by criteria to create the maximum electric field strength in the plane of the drying textile. We can describe this structure by means of an oriented graph, and we can also create a diagram of the EM waves inside this structure (Fig. 5)

By modifying the diagrams on the right side (Fig. 4) we can arrive at the resulting expression for calculating the E-field strength in the textile plane:

$E\left(\ell, \rho_{2}, \alpha_{\text {tex }}\right)=\sum_{n=0}^{\infty} \rho_{1}^{n}\left(\rho_{2}+\sigma_{2} e^{-x}\right)^{n} \cdot e^{-j \beta \ell(1+2 n)}$,

parameters $\rho_{2}$ and $\alpha_{\text {tex }}$ are given by the dielectric properties of the textile, so we can write the electric field strength dependent on relative permittivity $\varepsilon_{\text {tex }}$ and loss factor $\operatorname{tg} \delta_{\text {tex }}$ as follows

$E\left(\ell, \varepsilon_{\text {tex }}, \operatorname{tg} \delta_{\text {tex }}\right)=\frac{e^{\left(j \beta \ell+\alpha_{\text {tex }} \cdot t\right)}}{e^{\left(\alpha_{\text {tex }} \cdot t+2 j \beta \ell\right)}-\rho_{1} \rho_{2} \cdot e^{\alpha_{\text {tex }} \cdot t}-\rho_{1} \sqrt{1-\rho_{2}^{2}}}$.

Some examples of the application of this equation are given in Fig. 5. Electric field strength with respect to distance $\ell$ and to relative permittivity $\varepsilon_{\text {tex }}(\operatorname{tg} \delta=0.566)$.

As shown in Fig. 6, with decreasing permittivity the electric field strength in the textile increases, and the position of its maximum does not change. This finding is very important. During the drying process, the permittivity of the textile decreases. However, due to the increase in the electric field strength the resulting efficiency of the drying process does not decrease so quickly. Fig. 7 shows the dependence of the electric field strength on distance $\ell$ and $\operatorname{loss}$ factor $\operatorname{tg} \delta_{\text {tex }}\left(\varepsilon_{\text {tex }}\right.$ is constant). We can see that due to $\operatorname{tg} \delta_{\text {tex }}$ the electric field strength does not decrease significantly but changes the position of its maximum value.

During the drying process the quantity of water in the textile changes. We measured the complex permittivity of the textile with Agilent E4991A Material Analyzer (Fig. 9). Rela- 


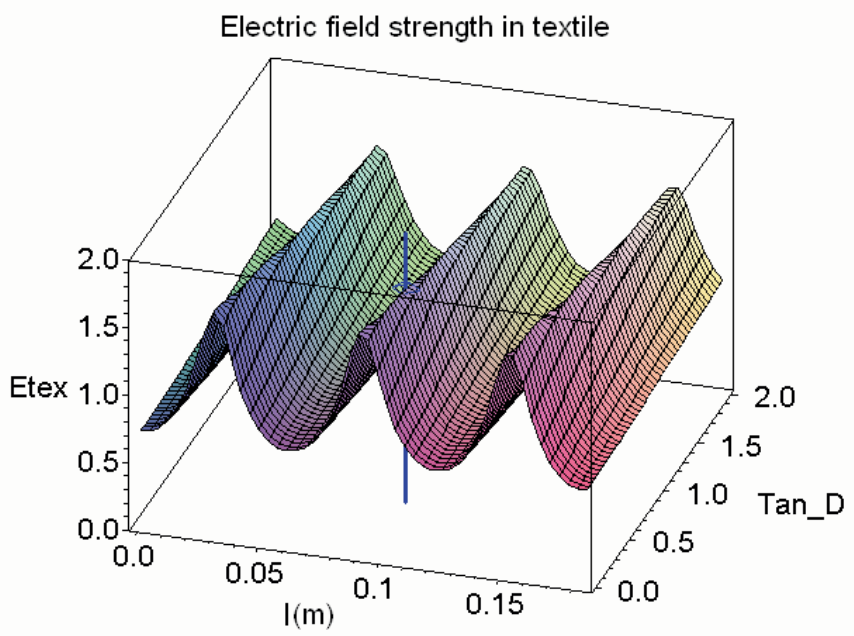

Fig. 7: Dependence of electric field strength on distance $\ell$ and loss factor $\operatorname{tg} \delta_{\text {tex }}\left(\varepsilon_{\text {tex }}=8\right)$

tive permittivity and loss factor are decreasing with decreasing water content. It can be seen in Fig. 8.

The next part presents the analysis in which the calculation with measured results of the permittivity and loss factor are used. Fig. 10 is a chart of the dependence of the electric field strength on distance for the case of a resonator without a textile (high peaks) and with a sample of wet textile (attenu-

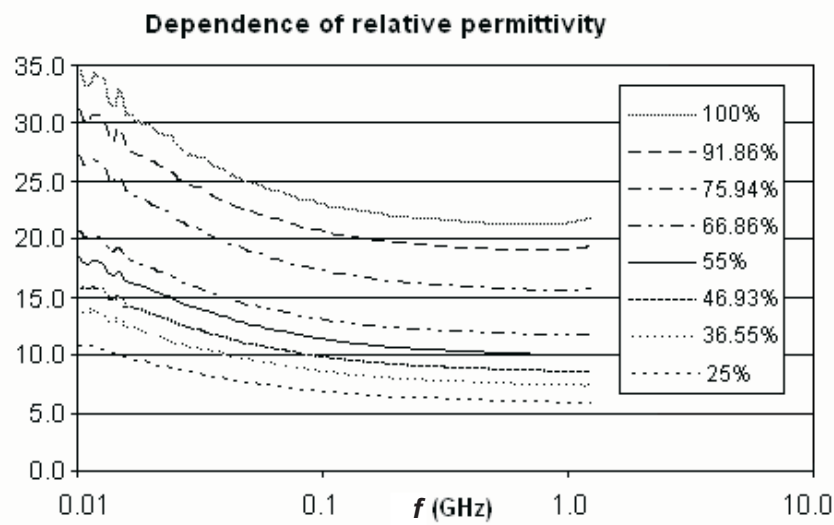

(a)

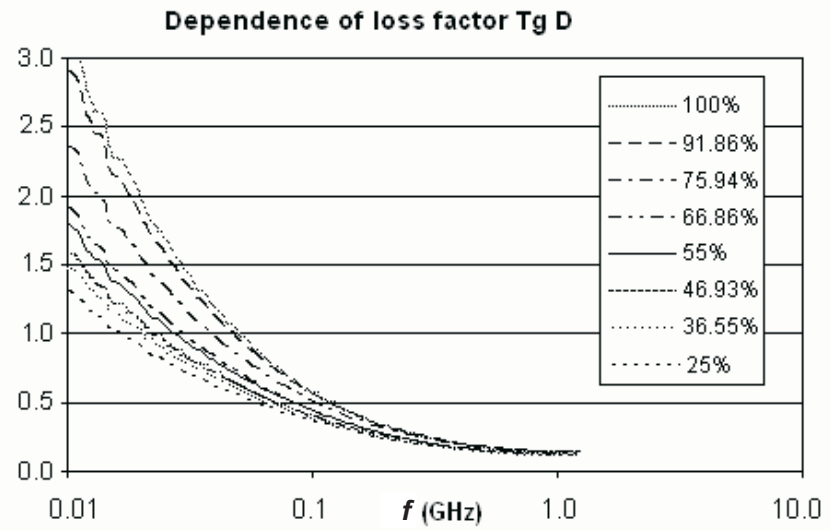

(b)

Fig. 8: Relative permittivity (a) and loss factor (b) are decreasing with frequency (horizontal axis) and with decreasing water content (set of parametric curves)

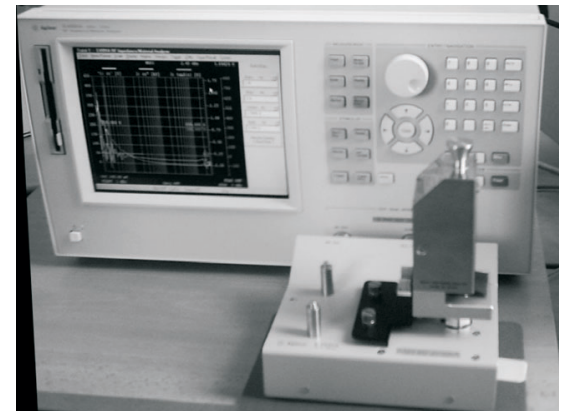

Fig. 9: Measurement system - the Agilent E4991A Material Analyzer

ated peaks). Fig. 11 shows a chart of the dependence of the electric field strength on distance for the case of four different volumes of moisture. We can see that the distance of the parallel plate and the textile changes in dependence on the moisture content.

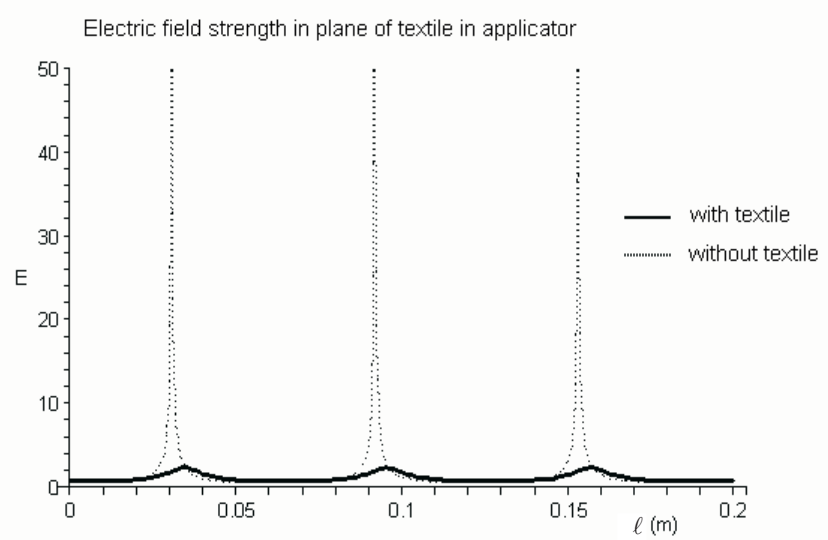

Fig. 10: Electric field strength in dependence on distance $\ell$ for the case of a resonator without textile (high peaks) and with a sample of wet textile

Distribution of Electric field strength in textile

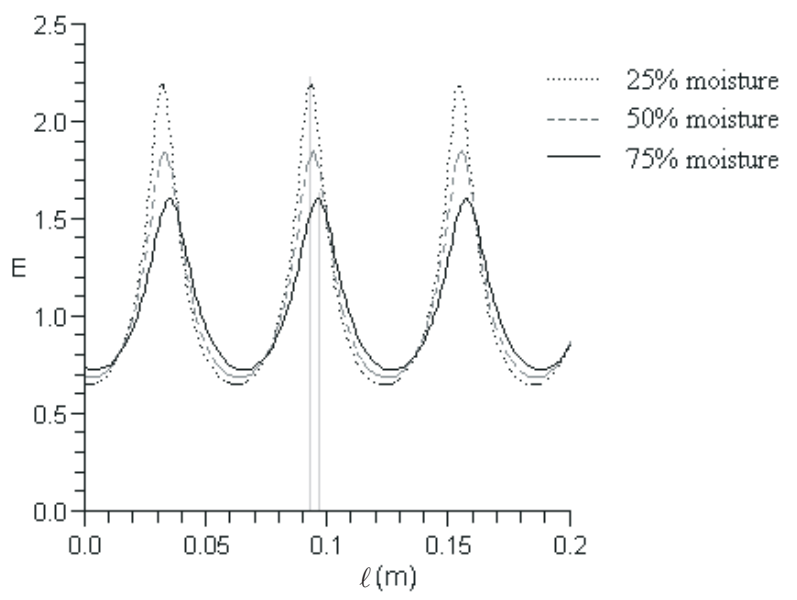

Fig. 11: Electric field strength in dependence on distance $\ell$ and moisture content (set of parametric curves)

\section{Realization of the applicator}

For our first experiments we built a single workable cell (Fig. 12), which was used for the first drying experiments (Fig. 3b, Fig. 4b) and for measurements of the drying curve. Fig. 13 shows the results of measurements of the moisture content in drying textile with respect to time. 


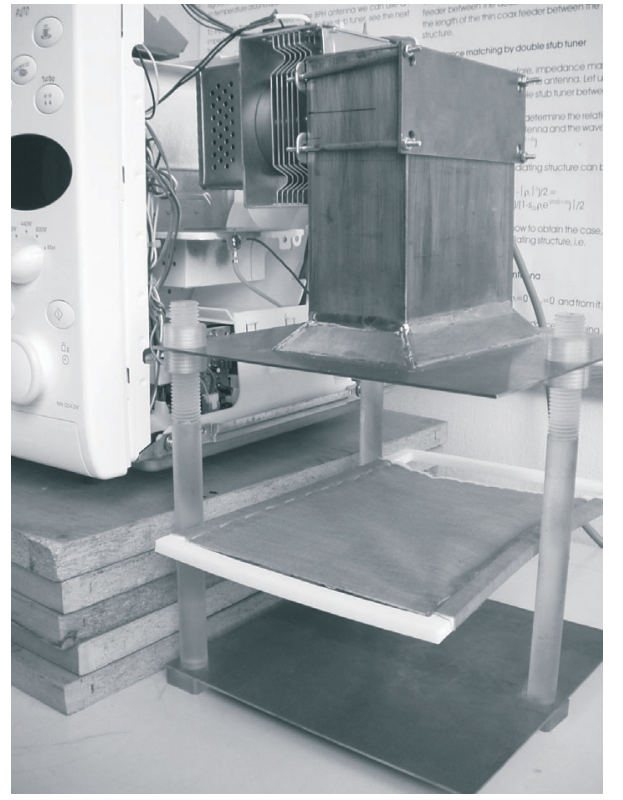

Fig. 12: Realization of a workable drying cell

Drying curve

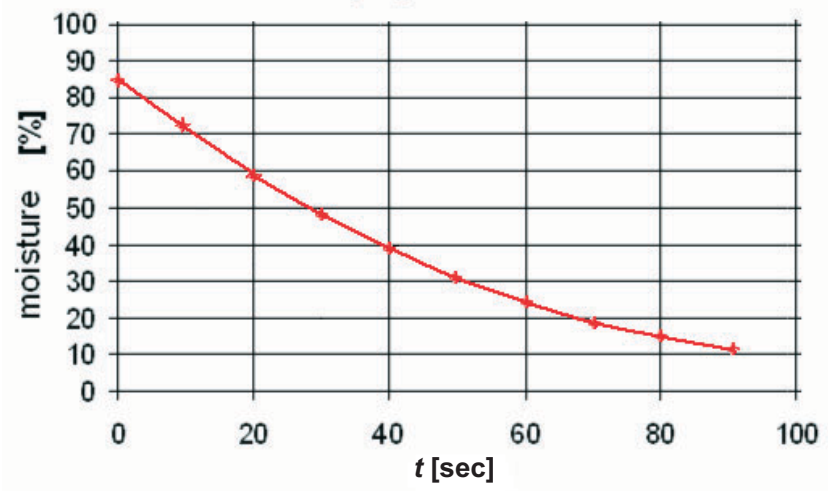

Fig. 13: Typical drying characteristic of this type applicator

Based on theoretical considerations developed at the Czech Technical University in Prague, a prototype of the microwave drying machine has been built in cooperation with the Research Institute of Textile Machines and the Technical University in Liberec, see Fig. 14a. The inside microwave part

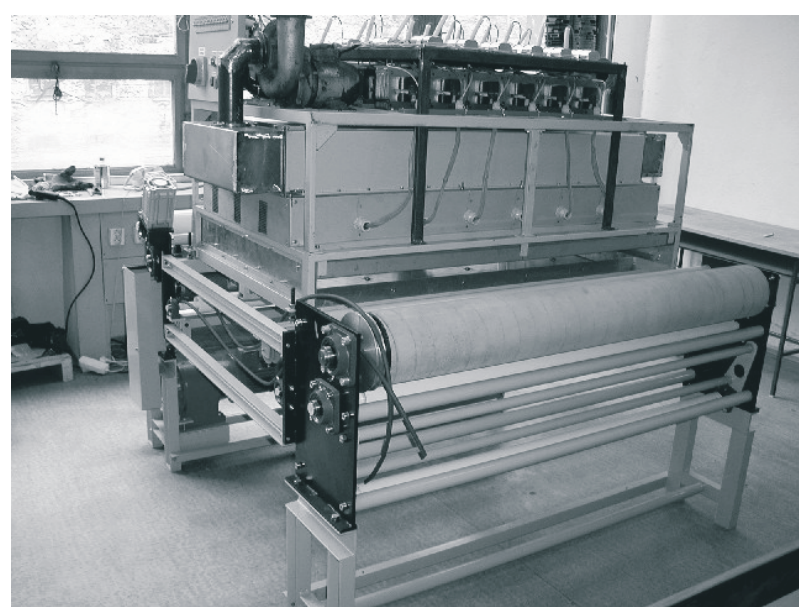

(a) of the system is shown in Fig. 14b. The waveguide horn apertures of several cells can be seen here (together with the tubes used for withdrawing the moisture). This apparatus was created with 17 heating cells: a matrix of 6 cells in the first row, then 5 cells in the second row, and again 6 cells in the third row. Each magnetron in all 17 cells delivers $1 \mathrm{~kW}$ at 2.45 GHz [3].

\section{Conclusion}

The novel results of our work are a description and a basic evaluation of a microwave open-resonant applicator for drying textile materials. Optimization of this applicator based on an analytical model is reported. The whole system is optimized, and also a single workable cell working at frequency $2.45 \mathrm{GHz}$.

\section{Acknowledgment}

This work has been supported by the Czech Grant Agency under grant 102/03/H086 Novel Approach and Coordination of Doctoral Education in Radioelectronics and Related Disciplines.

\section{References}

[1] Vrba, J.: Applications of Microwave Techniques. Prague: CTU Press 2001 (in Czech).

[2] Stejskal, M., Vrba, J., Klepl, R.: Microwave Drier for Fabrics. European Patent number EP1319914 A2 18-06-2003.

[3] Vrba, J.: Microwave Drying of Textile Materials. Prague: Series of Research Reports 2001-2004 (in Czech).

Ing. Marika Pourová

e-mail: pourovm@fel.cvut.cz

Prof. Ing. Jan Vrba, CSc.

e-mail:vrba@fel.cvut.cz

Department of Electromagnetic Field

Czech Technical University in Prague

Faculty of Electrical Engineering

Technická 2

16627 Prague 6, Czech Republic

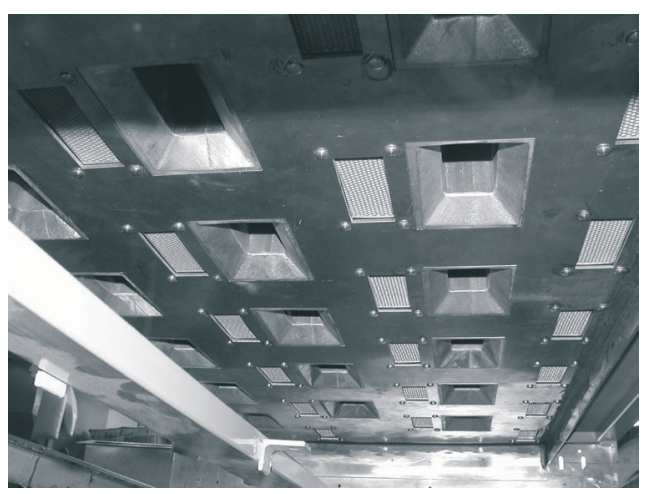

(b)

Fig. 14: Prototype of the drying machine (a) and inside microwave part of the system waveguide horn apertures (b) 